\title{
Blood Pressure During Endovascular Treatment Under Conscious Sedation or Local Anesthesia
}

Noor Samuels, MD, Rob A. van de Graaf, MD, Carlijn A.L. van den Berg, BSc, Daan Nieboer, MSc, Ismail Eralp, MD, PhD, Kilian M. Treurniet, MD, Bart J. Emmer, MD, PhD, Rogier V. Immink, MD, PhD, Charles B.L.M. Majoie, MD, PhD, Wim H. van Zwam, MD, PhD, Reinoud P.H. Bokkers, MD, PhD, Maarten Uyttenboogaart, MD, PhD, Boudewijn A.A.M. van Hasselt, MD, Jörg Mühling, MD, PhD, James F. Burke, MD, Bob Roozenbeek, MD, PhD, Aad van der Lugt, MD, PhD, Diederik W.J. Dippel, MD, PhD, Hester F. Lingsma, PhD, and Adriaan C.G.M. van Es, MD, PhD, on behalf of the MR CLEAN Registry Investigators Neurology ${ }^{\circledR}$ 2021;96:e171-e181. doi:10.1212/WNL.0000000000011006

\section{Abstract}

\section{Objective}

To evaluate the role of blood pressure (BP) as mediator of the effect of conscious sedation (CS) compared to local anesthesia (LA) on functional outcome after endovascular treatment (EVT).

\section{Methods}

Patients treated in the Multicenter Randomized Clinical Trial of Endovascular Treatment for Acute Ischemic Stroke in the Netherlands (MR CLEAN) Registry centers with CS or LA as preferred anesthetic approach during EVT for ischemic stroke were analyzed. First, we evaluated the effect of CS on area under the threshold (AUT), relative difference between baseline and lowest procedural mean arterial pressure $(\triangle \mathrm{LMAP})$, and procedural $\mathrm{BP}$ trend, compared to LA. Second, we assessed the association between BP and functional outcome (modified Rankin Scale $[\mathrm{mRS}]$ ) with multivariable regression. Lastly, we evaluated whether BP explained the effect of CS on mRS.

\section{Results}

In 440 patients with available BP data, patients treated under CS $(n=262)$ had larger AUTs (median 228 vs $23 \mathrm{~mm} \mathrm{Hg}^{*}$ min), larger $\triangle \mathrm{LMAP}$ (median 16\% vs 6\%), and a more negative BP trend $(-0.22$ vs $-0.08 \mathrm{~mm} \mathrm{Hg} / \mathrm{min})$ compared to LA $(\mathrm{n}=178)$. Larger $\triangle \mathrm{LMAP}$ and AUTs were associated with worse $\mathrm{mRS}$ (adjusted common odds ratio [acOR] per 10\% drop 0.87, 95\% confidence interval [CI] 0.78-0.97, and acOR per $\left.300 \mathrm{~mm} \mathrm{Hg} \mathrm{Hg}^{*} \mathrm{~min} 0.89,95 \% \mathrm{CI} 0.82-0.97\right)$. Patients treated under CS had worse mRS compared to LA (acOR 0.59, 95\% CI 0.40-0.87) and this association remained when adjusting for $\triangle \mathrm{LMAP}$ and AUT (acOR 0.62, 95\% CI $0.42-0.92$ ).

\section{Conclusions}

Large BP drops are associated with worse functional outcome. However, BP drops do not explain the worse outcomes in the CS group.

\author{
Correspondence \\ Dr. Samuels \\ n.samuels@erasmusmc.nl
}




\section{Glossary}

$\mathrm{a} \beta=$ adjusted $\beta ; \mathrm{acOR}=$ adjusted common odds ratio; ASPECTS = Alberta Stroke Program Early CT Score; AUT = area under the threshold; $\mathbf{B P}=$ blood pressure; $\mathbf{C I}=$ confidence interval; $\mathbf{C S}=$ conscious sedation; $\mathbf{e T I C I}=$ extended thrombolysis in cerebral infarction; EVT = endovascular treatment; GA = general anesthesia; IQR = interquartile range; LA = local anesthesia; LMAP = lowest procedural mean arterial pressure; MAP = mean arterial pressure; MR CLEAN = Multicenter Randomized Clinical Trial of Endovascular Treatment for Acute Ischemic Stroke in the Netherlands; mRS = modified Rankin Scale; NIHSS = NIH Stroke Scale; RCT = randomized controlled trial; $\mathbf{s I C H}=$ symptomatic intracranial hemorrhage.

Post hoc analyses of the Multicenter Randomized Clinical Trial of Endovascular Treatment for Acute Ischemic Stroke in the Netherlands (MR CLEAN) and the Highly Effective Reperfusion evaluated in Multiple Endovascular Stroke trials (HERMES) collaboration showed that general anesthesia (GA) is associated with worse clinical outcomes than nonGA. In these studies, non-GA was the composite of conscious sedation (CS) and local anesthesia (LA) at the groin puncture site only. ${ }^{1,2}$ Furthermore, among patients managed without GA, CS seemed to be associated with worse functional outcome compared to LA.,

Previous studies in patients receiving GA during endovascular treatment (EVT) reported worse outcomes in patients who experienced blood pressure (BP) drops during the procedure. ${ }^{5-9}$ The administration of anesthetic and analgesic agents may cause gradual or sudden declines in BP. This potentially impairs penumbra perfusion before recanalization. ${ }^{10-12}$ Considering that hypotension leads to worse outcomes in GA, hypotension might also contribute to worse outcomes in patients treated under CS or LA. There are limited data on BP measures during EVT among patients treated under CS or LA. ${ }^{13,14}$

In the present study, we explored the effect of CS on procedural $\mathrm{BP}$ and functional outcome, using patients under LA as control. In addition, we evaluated whether BP drops explain differences in functional outcome between anesthetic regimens.

\section{Methods}

\section{Study Population}

We used data from the MR CLEAN Registry, which is a prospective, multicenter, observational study including all patients who underwent EVT for ischemic stroke due to a large vessel occlusion in the Netherlands from March 2014 until November 2017. Detailed information on the description of variables and the methods of the MR CLEAN Registry have been reported previously. ${ }^{15}$ First, centers were excluded if they were non-MR CLEAN trial centers, did not perform EVT under CS or LA as the preferred anesthetic approach, or did not record periprocedural BP as part of protocol care. Second, patients were excluded when they were younger than 18 years, had an occlusion in the posterior circulation, or were treated after 6.5 hours of stroke onset. Third, we excluded patients who had no available BP data or were treated under GA as the initial anesthetic strategy during EVT in one of the centers with CS or LA as the preferred anesthetic approach.

To address the risk of bias through selective hemodynamic monitoring and BP data storage in patients at higher risk for hemodynamic instability, we additionally evaluated baseline characteristics of patients treated under CS and LA with and without BP data. Procedural BP values and administered medication were collected retrospectively from patients' records. Study results are reported in accordance with the Strengthening the Reporting of Observational Studies in Epidemiology (STROBE) statement. ${ }^{16}$

\section{Standard Protocol Approvals, Registrations, and Patient Consents}

The MR CLEAN Registry was approved by the medical ethics committee of the Erasmus University MC, Rotterdam, the Netherlands (MEC-2014-235). The institutional review board of each participating center approved the research protocol. At UMC Utrecht, additional approval to participate in the study was obtained from the local research board and ethics committee. The necessity of written informed consent was waived.

\section{Anesthetic Management}

To limit the risk of confounding by indication, only patients treated in centers that perform EVT under either CS or LA as the preferred anesthetic approach were selected. CS was defined as the administration of any sedative with or without analgesics (e.g., propofol, remifentanil) from 10 minutes before groin puncture until the time of recanalization, not requiring intubation. LA was defined as the use of LA (e.g., lidocaine) at the puncture site, without the use of any systemic analgesics or sedatives. Patients converted to GA during the procedure, defined as endotracheal intubation, were analyzed according to the initial anesthetic strategy to limit confounding by indication. The choice of anesthetic agents was at the discretion of the attending anesthesiologist or trained nurse. Anesthetic reports of all patients were reviewed for type, dosages, and time of administered anesthetic and vasoactive agents.

\section{Hemodynamic Management}

Standard hemodynamic monitoring included oxygen saturation, heart rate, noninvasive $\mathrm{BP}$, and temperature. Invasive $\mathrm{BP}$ monitoring was performed on an individual basis as determined by the anesthesiologist. The frequency of BP 


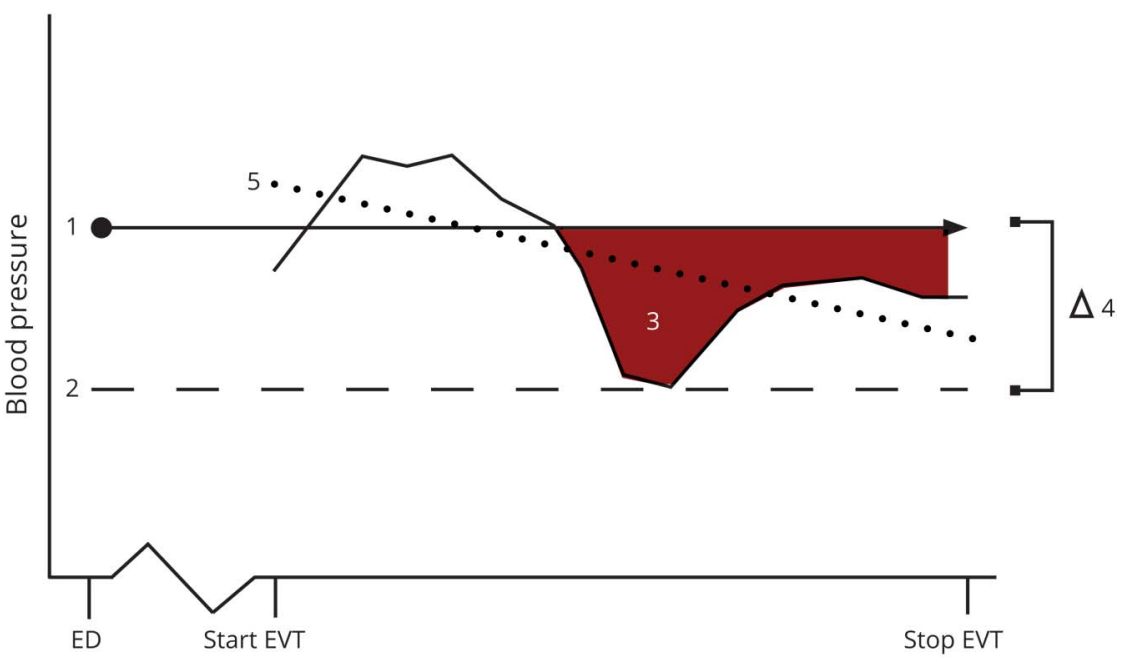

1. Mean arterial pressure (MAP) value on admission. 2. Lowest MAP. 3. Area under the threshold. 4. Relative difference between baseline MAP and lowest MAP ( $\triangle$ LMAP). 5. Average trend (slope). ED = emergency department; EVT = endovascular treatment. measurements depends on the local monitoring protocol. Systolic BP, diastolic BP, and mean arterial pressure (MAP) values, recorded between 10 minutes before groin puncture and time of recanalization, were retrieved from the patients' procedural anesthesia reports. Because there is no consensus on which BP-derived measures are most relevant and what should be avoided (e.g., drops, variability), we focused on 3 predefined orthogonal definitions that capture different elements of BP drops and variability ${ }^{17}$ : area under the threshold
(AUT, with MAP on admission as the threshold determined per patient) in $\mathrm{mm} \mathrm{Hg}^{*}$ minute, reflecting both the depth and duration of the relative hypotensive episode; the relative difference between the MAP on admission and the lowest MAP during the EVT procedure, expressed as percentage drop in MAP ( $\triangle \mathrm{LMAP})$, to account for shorter, larger BP drops; and the $\mathrm{BP}$ trend during the procedure, defined as the slope for each patient derived from a multilevel linear regression model with "time since start procedure" as a predictor, with a random

Figure 2 Flowchart of Patient Selection

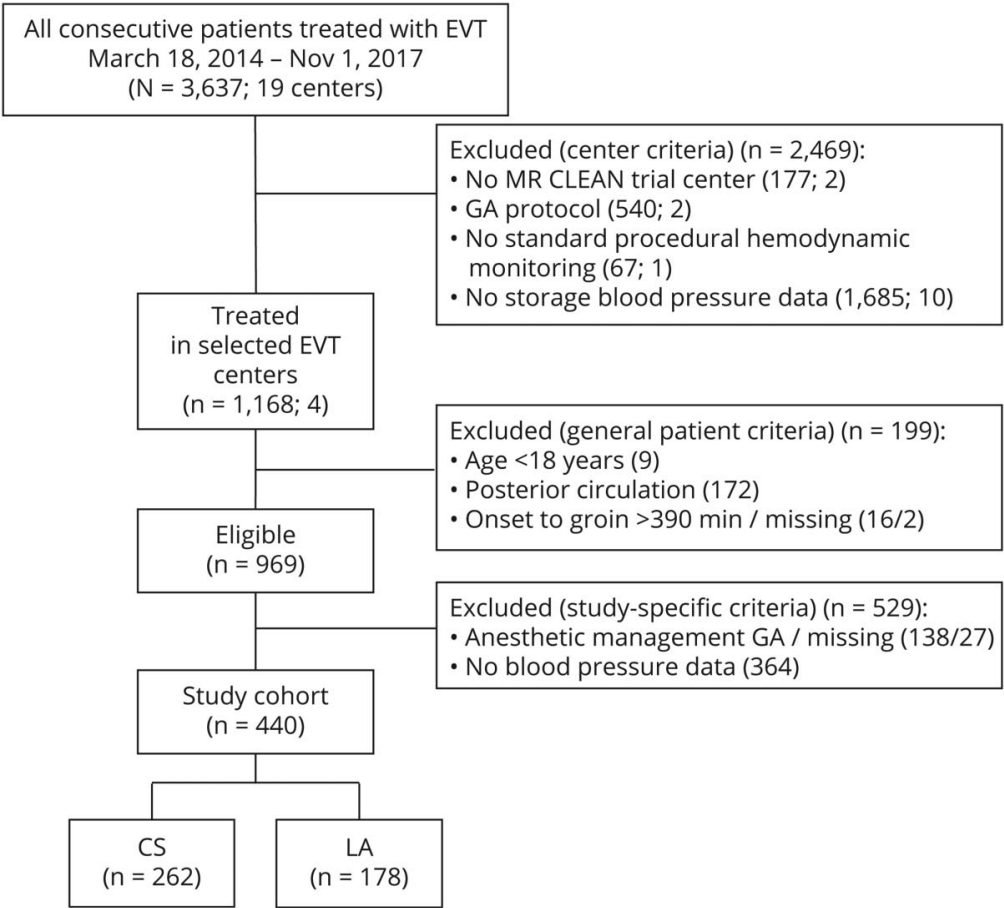

$\mathrm{CS}$ = conscious sedation; EVT = endovascular treatment; $\mathrm{GA}=$ general anesthesia; $\mathrm{LA}=$ local anesthesia; MR CLEAN = Multicenter Randomized Clinical Trial of Endovascular Treatment for Acute Ischemic Stroke in the Netherlands. 
Table 1 Baseline Characteristics

\begin{tabular}{|c|c|c|c|}
\hline & $\begin{array}{l}\text { CS } \\
(n=262)\end{array}$ & $\begin{array}{l}\text { LA } \\
(n=178)\end{array}$ & Missing \\
\hline \multicolumn{4}{|l|}{ Patient characteristics } \\
\hline Age, $y$ & $68(15)$ & $69(15)$ & \\
\hline Male sex & $128(49)$ & $103(58)$ & \\
\hline NIHSS & $16(11-19)$ & $15(11-19)$ & \\
\hline Left hemisphere & $118(45)$ & $97(55)$ & \\
\hline Systolic BP & $149(25)$ & $148(24)$ & \\
\hline Diastolic BP & $84(16)$ & $81(15)$ & \\
\hline IVT & $203(77)$ & $135(76)$ & \\
\hline \multicolumn{4}{|l|}{ Center } \\
\hline 1: Preferred approach $\mathrm{CS}^{\mathrm{a}}$ & $134(70)$ & $58(30)$ & \\
\hline 2: Preferred approach LA & $2(13)$ & $13(87)$ & \\
\hline 3: Preferred approach LA & $16(57)$ & $12(43)$ & \\
\hline 4: Preferred approach CS & $110(55)$ & $95(45)$ & \\
\hline \multicolumn{4}{|l|}{ Medical history } \\
\hline Previous stroke & $44(17)$ & $12(6.7)$ & $1 / 0$ \\
\hline Atrial fibrillation & $58(22)$ & $40(22)$ & $4 / 0$ \\
\hline Hypertension & $124(49)$ & $94(53)$ & $8 / 5$ \\
\hline Diabetes & $42(16)$ & $28(16)$ & $3 / 1$ \\
\hline Myocardial infarction & $29(11)$ & $24(14)$ & $6 / 1$ \\
\hline Prestroke mRS & & & $6 / 2$ \\
\hline 0 & $182(72)$ & $133(76)$ & \\
\hline 1 & $35(14)$ & $18(10)$ & \\
\hline 2 & $29(11)$ & $7(4.0)$ & \\
\hline$>2$ & $10(3.9)$ & $18(10)$ & \\
\hline \multicolumn{4}{|l|}{ Imaging } \\
\hline Occluded segment & & & $7 / 9$ \\
\hline M1 & $157(62)$ & $108(64)$ & \\
\hline M2 & $27(11)$ & $26(16)$ & \\
\hline ICA & $16(6.3)$ & $5(3.0)$ & \\
\hline ICA-T & $55(22)$ & $30(18)$ & \\
\hline ASPECTS & $9(8-10)$ & $9(8-10)$ & $6 / 9$ \\
\hline Collaterals & & & $9 / 14$ \\
\hline Absent & $14(5.5)$ & $9(5.5)$ & \\
\hline $\begin{array}{l}\text { Filling }<50 \% \text { of } \\
\text { occluded area }\end{array}$ & $97(38)$ & $63(38)$ & \\
\hline$\geq 50 \%$ but $<100 \%$ & 99 (39) & $65(40)$ & \\
\hline $\begin{array}{l}100 \% \text { of occluded } \\
\text { area }\end{array}$ & 43 (17) & $27(16)$ & \\
\hline
\end{tabular}

Table 1 Baseline Characteristics (continued)

$\begin{array}{ll}\text { CS } & \text { LA } \\ (n=262) & (n=178)\end{array} \quad$ Missing

Workflow, min

\begin{tabular}{llll}
\hline $\begin{array}{l}\text { Time from admission ED to } \\
\text { groin puncture }\end{array}$ & $41(28-69)$ & $44(30-73)$ & $12 / 7$ \\
\hline $\begin{array}{c}\text { Time from stroke onset to } \\
\text { groin puncture }\end{array}$ & 195 & 191 \\
& $(155-260)$ & $(155-244)$ & \\
\hline
\end{tabular}

Abbreviations: ASPECTS $=$ Alberta Stroke Program Early Computed Tomography Score; $\mathrm{BP}=$ blood pressure; $\mathrm{CS}=$ conscious sedation; $\mathrm{ED}=$ emergency department; ICA = internal carotid artery; ICA-T = internal carotid artery terminus; IVT = IV thrombolysis; LA = local anesthesia; M (segment) = middle cerebral artery; $\mathrm{mRS}=$ modified Rankin Scale; NIHSS = NIH Stroke Scale.

Values are $\mathrm{n}(\%)$; continuous data are presented as mean (SD) for normal distributed data or as median (interquartile range) for skewed data.

${ }^{\text {a }}$ Preferred approach changed in 2017 to LA.

slope to estimate patient-specific trends in BP measurements, for the continuous outcome systolic BP including a random effect for patient to account for within-patient variability (figure 1)..$^{7,8,18-20}$ Hemodynamic intervention was defined as the administration of any inotropes or vasopressors (e.g., ephedrine, phenylephrine) to increase BP or the use of sympathicolytics (e.g., labetalol, clonidine) to lower BP. BP was regulated according to institutional practices; in general, systolic BP was maintained between 140 and $185 \mathrm{~mm} \mathrm{Hg}$ with a diastolic BP below $105 \mathrm{~mm} \mathrm{Hg}$ based on anesthetic critical care recommendations. ${ }^{21}$

\section{Outcome Measures}

The primary outcome measure was score on the modified Rankin Scale (mRS). This is a 7-point scale ranging from 0 "no symptoms" to 6 "death," assessed at 90 days after EVT. ${ }^{22}$ Secondary outcomes included functional independence ( $\mathrm{mRS} \leq 2)$, mortality within 90 days post EVT, and NIH Stroke Scale (NIHSS) score indicating neurologic deficit at 24-48 hours after EVT. ${ }^{23}$ Procedure-related outcomes included occurrence of hemodynamic intervention, reperfusion grade, duration of the EVT procedure, and occurrence of procedure-related complications (i.e., vessel perforation, vessel dissection, new thrombus, distal thrombus, hemorrhage, and vasospasm). The reperfusion grade was assessed by the extended thrombolysis in cerebral infarction (eTICI) score on digital subtraction angiography, which ranges from 0 "no reperfusion or anterograde flow beyond site of occlusion" to 3 "complete reperfusion." ${ }^{24}$ Serious adverse events included symptomatic intracranial hemorrhage $(\mathrm{sICH}$, neurologic deterioration of $\geq 4$ points on the NIHSS, and a compatible hemorrhage on imaging assessed by an independent core laboratory according to the Heidelberg criteria), ${ }^{25}$ extracranial hemorrhage, neurologic deterioration (increase of $\geq 4$ points on the NIHSS), new ischemic stroke (imaging of new brain tissue infarction with any degree of corresponding neurologic deficit), and pneumonia. 
Procedure-related complications and eTICI scores were assessed by an independent core laboratory. Investigators who assessed primary and secondary outcomes were not aware of the type of anesthetic management during EVT.

\section{Statistical Methods}

Baseline characteristics of patients who underwent EVT under CS were compared with patients who received LA during the EVT procedure. Missing data were imputed using multiple imputations by chained equations based on relevant covariates. ${ }^{26}$

We tested 3 associations according to a 4-step approach. (1) We evaluated the effect of anesthetic modality on the predefined BP measures (i.e., AUT, $\triangle \mathrm{LMAP}$, and trend) and hemodynamic interventions during EVT with multivariable linear regression. We adjusted for age, sex, hypertension, diabetes, atrial fibrillation, history of myocardial infarction, previous stroke, systolic BP on admission, baseline NIHSS, prestroke mRS score, and treatment center. (2) We assessed the association between the predefined BP measures and functional outcome. This association was evaluated for all BP measures separately with ordinal logistic regression adjusted for age, sex, previous stroke, diabetes, atrial fibrillation, hypertension, history of myocardial infarction, prestroke mRS, baseline NIHSS, treatment with IV thrombolysis, Alberta Stroke Program Early CT Score (ASPECTS) at baseline, collateral score, time from stroke onset to recanalization, and treatment center. (3) We evaluated the effect of anesthetic modality on functional outcome using an ordinal logistic regression analysis. We adjusted for the following prognostic factors to account for potential imbalances between both anesthetic modalities: age, sex, previous stroke, diabetes, atrial fibrillation, hypertension, history of myocardial infarction, prestroke mRS score, baseline NIHSS, treatment with IV thrombolysis, ASPECTS at baseline, collateral score, time from stroke onset to recanalization, and treatment center. (4) To evaluate whether procedural BP explained the association between anesthetic modality and functional outcome, we additionally adjusted for the predefined BP measures that were associated with functional outcome based on multivariable analyses. We repeated step III for secondary outcomes (i.e., functional independence, mortality, early NIHSS, successful reperfusion, duration of procedure, serious adverse events, and procedure-related complications) using the appropriate regression analysis. Step IV was repeated for the secondary outcomes: functional independence, mortality, early NIHSS, and successful reperfusion.

To assess the association between predefined continuous BP measures and outcome, we compared a model containing restricted cubic splines for BP with a model including a linear $\mathrm{BP}$ term, based on the log likelihood ratio. Odds ratios for the association between BP and outcome were reported per $300 \mathrm{~mm} \mathrm{Hg}^{*}$ minutes for AUT or per $10 \%$ drop for $\Delta \mathrm{LMAP}^{7}$

The association between anesthetic approach and functional outcome could possibly be confounded by conversion from
LA to CS later on during the EVT procedure as patients who did worse during the procedure received CS later on, and therefore were likely to have worse functional outcome. For that reason, we performed a sensitivity analysis to compare patients receiving CS from the start $(<15$ minutes from start EVT) to patients who received LA from the beginning (this group is a composite of LA only and CS administration later on during the procedure, $>15$ minutes from EVT start). No correction for multiple testing was performed. Statistical analyses were performed with R 3.5.0 software (R Foundation for Statistical Computing, Vienna, Austria).

\section{Data Availability}

Data cannot be made available, as no patient approval has been obtained for sharing coded data. However, R syntax and output files of the analyses will be made available on request.

\section{Results}

From the 17 participating centers in the MR CLEAN Registry, only 4 centers collected BP data systematically according to protocol and reported LA or CS as the preferred anesthetic approach at start of the EVT (figure 2).

\section{Study Population}

Of the 969 eligible patients treated in 1 of the 4 centers with consistent periprocedural anesthetic management, we included 440 patients with available BP data, who underwent EVT for acute ischemic stroke due to large vessel occlusion, of whom $262 / 440(60 \%)$ received CS and 178/440 (40\%) received LA as procedural anesthetic strategy. Patients treated under CS were less often functionally dependent at presentation (prestroke $\mathrm{mRS}>2 ; 10 / 256,3.8 \%$ vs $18 / 176,10 \%$ ) but had a history of previous stroke (44/261 [17\%] vs 12/178 [6.7\%]) more often. Mean diastolic BP on admission was lower for patients receiving LA ( 81 [SD 15] vs 84 [SD 16] $\mathrm{mm} \mathrm{Hg}$; table 1). We did not find substantial differences in baseline characteristics between patients treated under LA with available BP data $(\mathrm{n}=178)$ and without $\mathrm{BP}$ data $(\mathrm{n}=$ 326). Also, no differences between patients treated under CS with available BP data $(\mathrm{n}=262)$ compared to patients treated under CS without BP data $(\mathrm{n}=38)$ were found.

\section{Procedural Management}

Average procedural systolic, diastolic, and mean arterial BPs were lower for patients who were treated under CS (figure 3 and table 2). AUT and $\triangle \mathrm{LMAP}$ were larger in the CS group (median AUT $228 \mathrm{~mm} \mathrm{Hg}^{*}$ min [interquartile range (IQR) 16-790] vs $23 \mathrm{~mm} \mathrm{Hg}^{*} \mathrm{~min}$ [0-200] and median $\triangle \mathrm{LMAP}$ $16 \%[5-31]$ vs $6 \%[0-16])$. Procedural systolic BP trend was more negative in patients treated under CS compared to LA $(-0.22 \mathrm{~mm} \mathrm{Hg}$ [SD 0.39] vs $-0.08 \mathrm{~mm} \mathrm{Hg}$ [SD 0.27]). BP elevating medications were administered more often in the CS group than the LA group $(59 / 262$ [23\%] vs 6/178 [3.4\%]). BP-lowering medication was administered in $15 / 262$ (5.7\%) patients in the CS group and in 7/178 (3.9\%) patients 

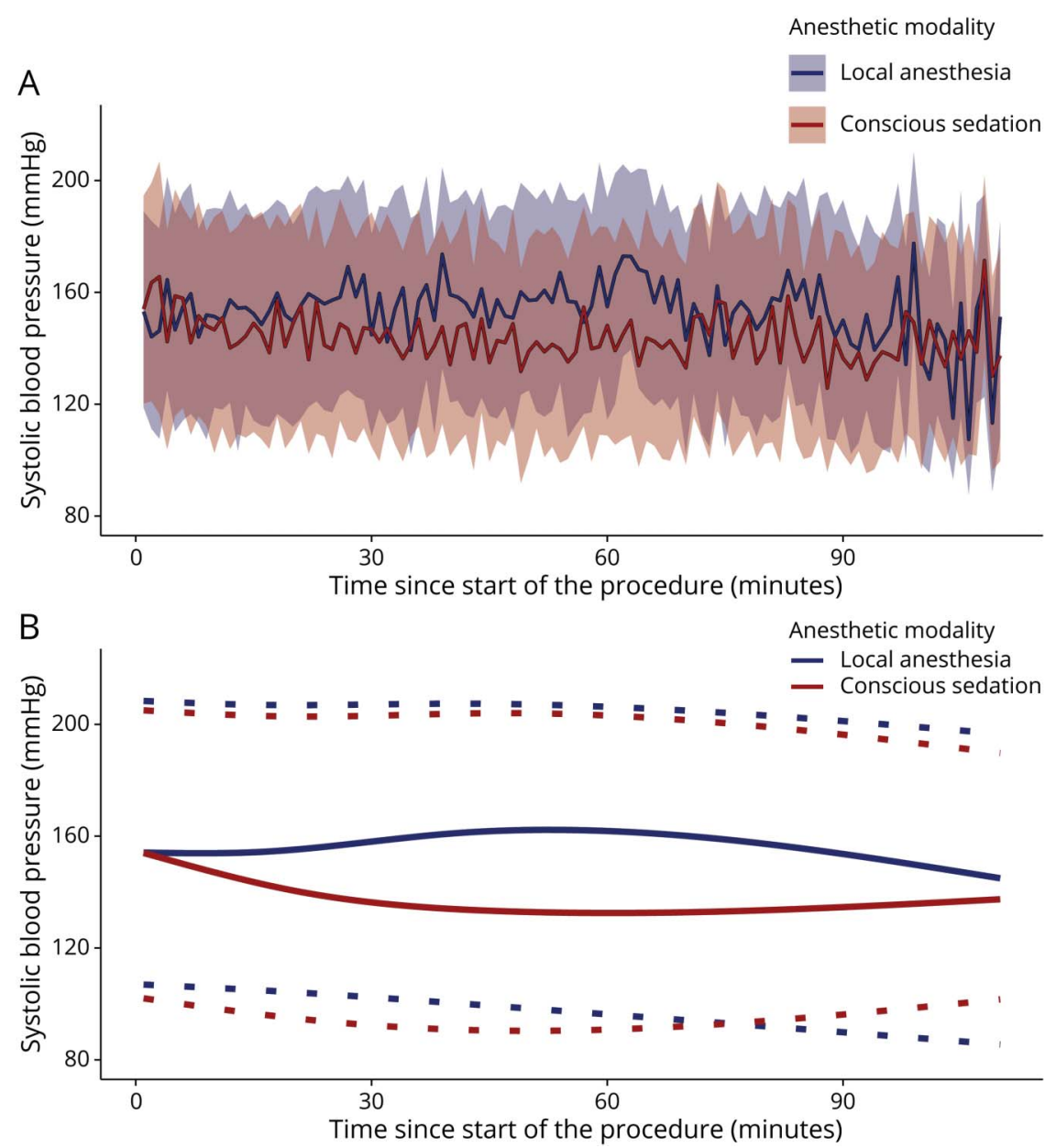

(A) Nonsmoothed mean systolic BP curves for both anesthetic modalities with $95 \%$ tolerance interval (band). (B) Smoothed mean systolic BP curves during EVT procedure for both anesthetic modalities (continuous line) with 95\% tolerance interval (dotted line).

in the LA group. Analgesics were used in 223/262 (85\%) patients in the CS group, of which remifentanil was administered most often $(116 / 262[44 \%])$. Sedatives were administered in $142 / 262$ (54\%) patients, of which propofol was used most frequently (127/262 [48\%]) (table 2). Conversion to GA requiring intubation occurred in 3 patients in the CS group and in 3 patients in the LA group.

\section{Association Between Anesthetic Management and Procedural BP}

CS was associated with larger AUTs (adjusted $\beta$ [a $\beta] 368$ [95\% confidence interval (CI) 242 to 494]) and larger $\triangle$ LMAP (a 8.1 [95\% CI 4.9 to 11.4]) compared to LA based on multivariable linear regression. Furthermore, CS was associated with a more decreasing procedural systolic BP trend (a $\beta-0.14[95 \% \mathrm{CI}-0.21$ to -0.07$]$ ).

\section{Association Between Procedural BP and Outcome}

Both $\triangle$ LMAP (adjusted common odds ratio [acOR] 0.89 per $10 \%$ drop from baseline [95\% CI $0.80-0.99]$ ) and AUT (acOR 0.89 per $300 \mathrm{~mm} \mathrm{Hg}^{*} \min$ [95\% CI 0.82-0.96]) were associated with a shift towards worse functional outcome in multivariable analysis. Procedural BP trend was not associated with functional outcome (acOR 0.85 per $\mathrm{mm} \mathrm{Hg}$ per minute [95\% CI 0.51-1.43]).

\section{Association Between Anesthetic Management and Outcome}

Patients undergoing EVT for acute ischemic stroke under CS were more likely to have poor mRS scores at 90 days compared to LA (acOR 0.59 [95\% CI 0.40-0.87]; table 3 and figure 4). The sensitivity analysis, comparing patients receiving CS from the beginning of the procedure $(n=51)$ to patients receiving LA from the beginning of the procedure ( $\mathrm{n}$ = 389) (acOR 0.49 [95\% CI 0.26-0.91]), obtained similar results to the primary analysis comparing CS administration at any time point during the procedure to LA. Functional independence at 90 days was less often seen in patients who underwent CS compared to LA (aOR 0.49 [95\% CI $0.30-0.83]$ ). There were no differences in all-cause mortality (aOR 1.78 [95\% CI 0.96-3.02]), NIHSS at 24-48 hours postEVT (a 1.13 [95\% CI -0.38 to 2.64]), and successful reperfusion grades (aOR 1.01 [95\% CI 0.66-1.65]) between groups. Procedure duration was almost 20 minutes longer in 
Table 2 Procedural Anesthetic and Hemodynamic Data

\begin{tabular}{|c|c|c|}
\hline & $\operatorname{CS}(n=262)$ & LA $(n=178)$ \\
\hline \multicolumn{3}{|l|}{ Medication $^{a}$} \\
\hline \multicolumn{3}{|l|}{ Muscle relaxant } \\
\hline Rocuronium & $3(1.1)$ & $2(1.1)$ \\
\hline Inotropes/vasopressors & $59(23)$ & $6(3.4)$ \\
\hline Atropine & $17(6.5)$ & $1(0.6)$ \\
\hline Ephedrine & $16(6.1)$ & $3(1.7)$ \\
\hline Epinephrine & $2(0.8)$ & 0 \\
\hline Isoprenaline & $2(0.8)$ & 0 \\
\hline Norepinephrine & $20(7.6)$ & $3(1.7)$ \\
\hline Phenylephrine & $24(9.2)$ & $2(1.1)$ \\
\hline Sympatholytics & $15(5.7)$ & $7(3.9)$ \\
\hline Clonidine & $1(0.4)$ & $4(2.2)$ \\
\hline Ketanserine & 0 & $1(0.6)$ \\
\hline Labetalol & $8(3.1)$ & $2(1.1)$ \\
\hline Nimodipine & $6(2.3)$ & 0 \\
\hline Urapidil & 0 & $1(0.6)$ \\
\hline Analgesics & $223(84)$ & - \\
\hline Alfentanil & $49(19)$ & - \\
\hline Fentanyl & $11(4.2)$ & - \\
\hline Morphine & $1(0.4)$ & - \\
\hline Remifentanil & $116(44)$ & - \\
\hline Sufentanil & $46(18)$ & - \\
\hline Sedatives & $142(53)$ & \\
\hline Esketamine & $12(4.6)$ & - \\
\hline Midazolam & $8(3.1)$ & - \\
\hline Propofol & $127(48)$ & - \\
\hline \multicolumn{3}{|c|}{ Blood pressure values, $\mathrm{mm} \mathrm{Hg}$} \\
\hline SBP & $141(123-164)$ & $155(135-173)$ \\
\hline DBP & $76(67-84)$ & $80(70-92)$ \\
\hline MAP & $100(89-115)$ & $107(94-121)$ \\
\hline$\triangle$ LMAP $^{\mathbf{b}}$ & $16(5.2-31)$ & $6.0(0-16)$ \\
\hline AUT, mm Hg*min & $228(16-790)$ & $23(0-200)$ \\
\hline Trend SBP ${ }^{c}$ & $-0.22(0.39)$ & $-0.08(0.27)$ \\
\hline
\end{tabular}

Abbreviations: AUT = area under threshold; CS = conscious sedation; DBP = diastolic blood pressure; $\mathrm{LA}=$ local anesthesia; $\triangle \mathrm{LMAP}=$ relative difference baseline mean arterial pressure and lowest procedural mean arterial pressure; MAP = mean arterial pressure; SBP = systolic blood pressure.

Values are $\mathrm{n}(\%)$, mean (SD), or median (interquartile range).

a Percentages may add up to more than 100 owing to combined administration of medication.

b Percentage drop from baseline MAP.

c $\beta$ coefficient. the CS group compared to the LA group (median 70 [44-90] vs 51 [33-74] minutes). The occurrence of procedure-related complications did not differ between patients treated under CS and LA (9/262 [3\%] vs 5/178 [4\%]; aOR 1.45 [95\% CI $0.89-2.31])$.

\section{Effect of BP on the Association Between Anesthetic Management and Outcome}

Additional adjustment for $\triangle \mathrm{LMAP}$ and AUT did not explain the association between anesthetic modality and functional outcome (acOR 0.62 [95\% CI 0.42-0.92]; table 3). $\triangle$ LMAP and AUT did not explain the association between anesthetic modality and any of the secondary outcomes.

\section{Discussion}

In this study, we evaluated the effect of CS on procedural hypotension, BP trend, and hemodynamic interventions compared to LA. Second, we assessed whether there was an association between the 3 predefined BP measures and outcomes. Third, we evaluated the effect of CS on functional outcome compared to LA. Finally, we explored whether the effect of anesthetic management on outcomes could be explained by procedural hypotension or BP trend. We found that $\mathrm{CS}$ was associated with more $\mathrm{BP}$ drops and that these BP drops were related to worse outcomes. However, the BP drops did not explain the effect of CS on functional outcome compared to LA.

Similar to previous studies, we found that patients treated under CS had lower average procedural BP and more BP drops compared to patients treated under LA. Consequently, more hemodynamic interventions were required to increase $\mathrm{BP}$ in patients treated under $\mathrm{CS} .^{7,13,27}$

A drop in MAP from baseline and larger AUT were independently associated with worse functional outcome. Similar, previous studies reported worse functional outcomes in patients with a drop in MAP from baseline of $\geq 10 \%$ who received CS or GA during the procedure. ${ }^{14,19,28}$ A recent study found that larger AUTs were associated with worse functional outcome in patients receiving GA as well as in patients receiving monitored anesthesia care, which is a composite of CS and LA. ${ }^{7}$ In our study, BP drops were relatively mild, especially in the LA group, compared to what has been observed in patients treated under GA (median AUT in our LA group of $23 \mathrm{~mm} \mathrm{Hg}^{*}$ min [0-200] vs $984 \mathrm{~mm} \mathrm{Hg}^{*} \min [227-1,968]$ in patients treated under GA and median $\triangle \mathrm{LMAP}$ in our LA group of $6 \%$ [0-16] vs 39\% [23-49] in patients treated under GA). ${ }^{7,8,28}$ The small hemodynamic variability observed in patients treated under LA underlines the importance of including LA as a treatment arm besides CS and GA in future randomized controlled trials (RCTs) focusing on optimal anesthetic and hemodynamic management during EVT.

In this study, patients treated under CS had worse functional outcome compared to patients treated under LA. Hypotension and 
Table 3 Effect of Conscious Sedation (CS) vs Local Anesthesia (LA) on Outcomes, Unadjusted (model A), Adjusted for Potential Confounding Variables (model B), and With Additional Adjustment for Blood Pressure (model C)

\begin{tabular}{|c|c|c|c|c|c|}
\hline & $\operatorname{CS}(n=262)$ & LA $(n=178)$ & $\begin{array}{l}\text { A: Unadjusted effect, } \\
\text { CS vs LA, (c)OR }(95 \% \mathrm{Cl})\end{array}$ & $\begin{array}{l}\text { B: Adjusted effect, CS vs } \\
\text { LA, a(c)OR }(95 \% \mathrm{Cl})\end{array}$ & $\begin{array}{l}\text { C: Adjusted effect, including } \\
\Delta \text { LMAP }^{\mathrm{a}} \text { and AUT, }{ }^{\mathrm{b}} \text { CS vs LA, } \\
\text { a(c)OR }(95 \% \mathrm{CI})\end{array}$ \\
\hline \multicolumn{6}{|l|}{ Primary outcome } \\
\hline mRS at 90 days & $4(2-6)$ & $3(1-4)$ & $0.56(0.40-0.79)$ & $0.59(0.40-0.87)$ & $0.62(0.42-0.92)$ \\
\hline \multicolumn{6}{|l|}{$\begin{array}{l}\text { Secondary outcomes, } \\
\text { clinical }\end{array}$} \\
\hline $\mathrm{mRS} \leq 2$ at 90 days & $80(34)$ & $82(50)$ & $0.53(0.36-0.78)$ & $0.49(0.30-0.83)$ & $0.53(0.30-0.85)$ \\
\hline Mortality at 90 days & $70(29)$ & $33(20)$ & $1.51(0.95-2.37)$ & $1.78(0.96-3.02)$ & $1.70(0.95-3.18)$ \\
\hline NIHSS 24-48 hours & $10(4-16)$ & $8(3-15)$ & $1.68(0.05-3.31)^{c}$ & $1.13(-0.38-2.64)^{c}$ & $0.88(-0.67-2.43)^{c}$ \\
\hline \multicolumn{6}{|l|}{$\begin{array}{l}\text { Secondary outcome, } \\
\text { radiologic }\end{array}$} \\
\hline $\begin{array}{l}\text { Successful reperfusion } \\
\text { after intervention } \\
(\mathrm{e} T I C I \geq 2 \mathrm{~B})\end{array}$ & $175(69)$ & $122(70)$ & $0.96(0.64-1.46)$ & $1.01(0.66-1.65)$ & $1.11(0.70-1.81)$ \\
\hline
\end{tabular}

Secondary outcomes,

workflow

\begin{tabular}{lllll}
\hline Duration of procedure & $70(44-90)$ & $51(33-74)$ & $15.9(9.49-22.2)^{c}$ & $14.3(8.17-20.50)^{c, d}$ \\
\hline $\begin{array}{l}\text { Secondary outcomes, } \\
\text { safety measures }\end{array}$ & $9(4)$ & $5(3)$ & $1.57(1.01-2.45)$ & $1.45(0.89-2.31)$ \\
\hline $\begin{array}{l}\text { Procedure-related } \\
\text { complications }\end{array}$ & $13(5.0)$ & $4(2.3)$ & $2.27(0.79-8.17)$ & $2.74(0.87-10.4)$ \\
\hline Symptomatic ICH & $5(1.9)$ & $7(3.9)$ & $0.48(0.14-1.51)$ & $0.52(0.13-1.98)$ \\
\hline ECH & $18(6.9)$ & $8(4.5)$ & $1.57(0.69-3.90)$ & $1.49(0.57-4.14)$ \\
\hline $\begin{array}{l}\text { Neurologic } \\
\text { deterioration }\end{array}$ & $7(2.7)$ & $2(1.1)$ & $2.42(0.58-16.3)$ & $4.80(0.84-20.1)$ \\
\hline New ischemic stroke & $28(11)$ & $16(9.0)$ & $1.21(0.64-2.36)$ & $1.04(0.50-2.23)$ \\
\hline Pneumonia & & & & \\
\hline
\end{tabular}

Abbreviations: $\mathrm{acOR}=$ adjusted common odds ratio; $\mathrm{AUT}=$ area under threshold; $\mathrm{Cl}=$ confidence interval; $\mathrm{ECH}=$ extracranial hemorrhage; $\mathrm{eTICI}=$ extended thrombolysis in cerebral infarction; ICH = intracranial hemorrhage; $\triangle \mathrm{LMAP}=$ relative difference, baseline mean arterial pressure and lowest procedural mean arterial pressure; $\mathrm{mRS}=$ modified Rankin Scale; NIHSS = NIH Stroke Scale.

Values are $\mathrm{n}(\%)$ or median (interquartile range). A: Univariable regression analyses; B: multivariable regression analyses (adjusted for age, sex, baseline NIHSS, prestroke mRS, history of stroke, hypertension, diabetes, atrial fibrillation, myocardial infarction, IV thrombolysis, Alberta Stroke Program Early CT Score score at baseline, time between stroke onset and recanalization, center): C: multivariable regression analyses (adjusted for the same variables as in step

2 with an additional adjustment for $\triangle$ LMAP and AUT to evaluate whether hypotension explains the effect of CS on outcome, i.e., reduces the effect estimate).

a Per $10 \%$ drop.

${ }^{\mathrm{b}}$ Per $300 \mathrm{~mm} \mathrm{Hg*}$ minute.

${ }^{\mathrm{C}}$ Reported effect measure is $\beta$ coefficient.

${ }^{\mathrm{d}}$ Adjustment for time between stroke onset and groin puncture instead of time between stroke onset and recanalization.

procedural BP trend did not explain the negative association of CS with functional outcome in our study. Because there were no large differences in baseline characteristics between patients treated under CS and LA, including neurologic deficit according to the NIHSS at baseline, adjustments for potential covariates did not reduce the effect of CS on outcome compared to LA. Therefore, the effect of CS on functional outcome might be caused by confounders not accounted for in the analyses. The decision to perform EVT under CS is likely to be made by the treating interventionalist and anesthesiologist based on clinical measures not reflected by the NIHSS score, for example patient agitation and motion. Furthermore, the NIHSS performed in an acute and time-restrained clinical situations might less well comprise mild to moderate neglect, disorientation, and aphasia, which could be the determinants of the anesthetic approach. Previous trials reported equivalent functional outcome among patients treated under GA or CS, which is likely due to the strict hemodynamic regimens as part of the anesthetic protocols. ${ }^{29-31}$ A pooled analysis of these RCTs suggested that worse outcome after EVT might be associated with BP variability instead of the anesthetic strategy itself. However, conclusions of this study were restricted to the association between BP variability and neurologic outcomes, stratified by anesthetic modality. ${ }^{28}$

In several EVT-capable centers with CS or LA as the preferred anesthetic approach during EVT, the involvement of the anesthesiologist is limited to patients who are hemodynamically 


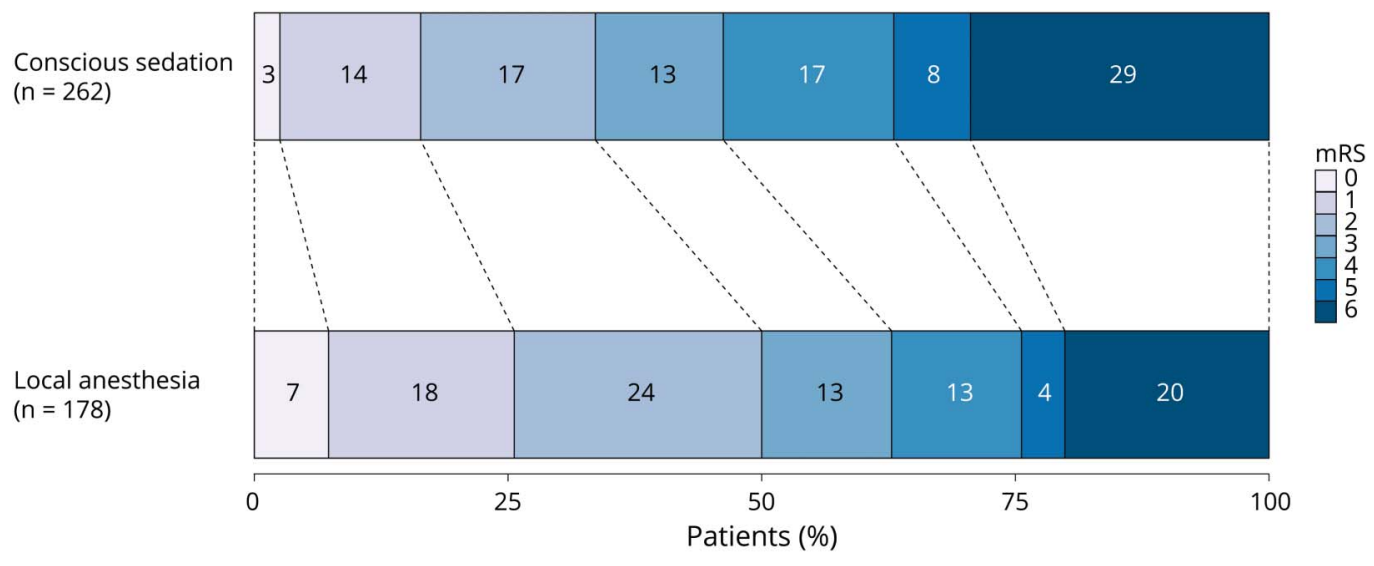

unstable or require GA. Because these results suggest that BP drops and hemodynamic interventions are seen during both CS and LA, hemodynamic monitoring and rapid treatment of hemodynamic instability during EVT should not be restricted to patients treated under GA only.

Our study has several limitations. First, due to the retrospective observational design of this study, results could have been confounded by variables not adjusted for in the analyses. Patients who are more affected at presentation are more likely to receive CS and hemodynamic monitoring, meaning residual confounding is present in this cohort. To limit the risk of confounding by indication, we performed a sensitivity analysis for patients who received sedatives or analgesics from the beginning of the procedure. In the sensitivity analysis among patients who received CS from the beginning of the EVT procedure compared to patients receiving LA from the beginning, a similar effect of CS on outcome was found. This suggests that conversion from LA to CS was not directly related to patient status at baseline and confounding by indication might be less likely. Furthermore, although we selected centers reporting either CS or LA as the preferred approach, we observed that a significant number of patients received the nonpreferred initial anesthetic approach. Because we selected centers with CS or LA as the preferred anesthetic approach and standard hemodynamic monitoring, the generalizability of our findings to patients treated under different anesthetic or hemodynamic regimens is limited. Second, there is no consensus on how to quantify procedural hypotension and BP variability. A different quantification of procedural hemodynamics could alter the effect of anesthetics on outcome. Lastly, as heterogeneity in anesthetic approach definitions exists, comparability is difficult as sedation is a continuum ranging from minimal to deep sedation, with a concomitant variety in physiologic effects (e.g., arterial hypotension, bradycardia, respiratory depression).

Hemodynamic interventions to maintain hemodynamic stability are common during EVT under CS and LA. In a cohort of patients treated with EVT under strict BP management, decreases in BP are small and do not explain the differences in functional outcome between patients treated under CS and LA. As BP drops by means of $\triangle \mathrm{LMAP}$ and AUT are independently associated with worse functional outcome, we advocate to monitor and avoid BP drops (i.e., ensure hemodynamic stability) during EVT. Further randomized controlled trials are needed to determine whether hemodynamic interventions improve patient outcomes.

\section{Study Funding}

The authors received no funding for this study. The MR CLEAN Registry is partially funded by unrestricted grants from Toegepast Wetenschappelijk Instituut voor Neuromodulatie, Twente University (TWIN), Erasmus MC University Medical Center, Maastricht University Medical Center, and Amsterdam University Medical Center. The study was additionally funded by the European Union's Horizon 2020 research and innovation program under grant agreement 777072 (In Silico Trials for Treatment of Acute Ischemic Stroke [INSIST]), which played no role in study design, patient enrollment, data collection, analysis, or writing of the manuscript. Erasmus $\mathrm{MC}$ received compensation from Stryker, Medtronic, and Bracco Imaging Ltd for activities of A.v.d.L. and D.W.J.D as a consultant. Amsterdam University Medical Center received funds from Stryker for consultations by C.B.L.M.M. Maastricht University Medical Center received funds from Stryker and Codman for consultations by W.H.v.Z.

\section{Disclosure}

A. van der Lugt and D.W.J. Dippel are the recipients of unrestricted grants from Dutch Heart Foundation, Dutch Brain Foundation, The Netherlands Organisation for Health Research and Development, Health Holland Top Sector Life Science, AngioCare BV, Covidien/EV3, MEDAC GmbH/ LAMEPRO, Top Medical/Concentric, Thrombolytic Science LLC, Stryker, Medtronic and Penumbra Inc. for the conduct of trials of acute treatment for stroke. C.B.L.M. Majoie 
received funds from TWIN Foundation and European Commission (related to this project, paid to institution) and from CVON/Dutch Heart Foundation, Stryker, Health Evaluation Netherlands (unrelated; all paid to institution), and is shareholder of Nico.lab, a company that focuses on the use of artificial intelligence for medical imaging analysis. $\mathrm{N}$. Samules, R.A. van de Graaf, C.A.L. van den Berg, D. Nieboer, I. Eralp, K.M. Treurniet, B.J. Emmer, R.V. Immink, R.P.H. Bokkers, M. Uyttenboogaart, B.A.A.M. van Hasselt, J. Mühling, J.F. Burke, B. Roozenbeek, H.F. Lingsma, and A.C.G.M. van Es report no disclosures. Go to Neurology.org/ $\mathrm{N}$ for full disclosures.

\section{Publication History}

Received by Neurology April 10, 2020. Accepted in final form August 24, 2020.

Appendix 1 Authors

\begin{tabular}{lll}
\hline Name & Location & Contribution \\
\hline $\begin{array}{lll}\text { Noor Samuels, } \\
\text { MD }\end{array}$ & $\begin{array}{l}\text { Erasmus MC, } \\
\text { University Medical } \\
\text { Center, Rotterdam }\end{array}$ & $\begin{array}{l}\text { Study concept and design, } \\
\text { data acquisition, statistical } \\
\text { analyses, drafting the } \\
\text { manuscript }\end{array}$ \\
\hline
\end{tabular}

Rob A. van de Erasmus MC, Study concept and design Graaf, MD University Medical data acquisition, statistical Center, Rotterdam analyses, critical revision of the manuscript for intellectual content

\begin{tabular}{|c|c|c|}
\hline $\begin{array}{l}\text { Carlijn A.L. van } \\
\text { den Berg, BSc }\end{array}$ & $\begin{array}{l}\text { Erasmus MC, } \\
\text { University Medical } \\
\text { Center, Rotterdam }\end{array}$ & $\begin{array}{l}\text { Major role in data } \\
\text { acquisition, statistical } \\
\text { analyses, critical revision of } \\
\text { the manuscript for } \\
\text { intellectual content }\end{array}$ \\
\hline $\begin{array}{l}\text { Daan Nieboer, } \\
\text { MSc }\end{array}$ & $\begin{array}{l}\text { Erasmus MC, } \\
\text { University Medical } \\
\text { Center, Rotterdam }\end{array}$ & $\begin{array}{l}\text { Statistical analyses, critical } \\
\text { revision of the manuscript } \\
\text { for intellectual content }\end{array}$ \\
\hline $\begin{array}{l}\text { Ismail Eralp, MD, } \\
\text { PhD }\end{array}$ & $\begin{array}{l}\text { Erasmus MC, } \\
\text { University Medical } \\
\text { Center, Rotterdam }\end{array}$ & $\begin{array}{l}\text { Critical revision of the } \\
\text { manuscript for intellectual } \\
\text { content }\end{array}$ \\
\hline $\begin{array}{l}\text { Kilian M. } \\
\text { Treurniet, MD }\end{array}$ & $\begin{array}{l}\text { Amsterdam UMC, } \\
\text { University of } \\
\text { Amsterdam }\end{array}$ & $\begin{array}{l}\text { Critical revision of the } \\
\text { manuscript for intellectual } \\
\text { content }\end{array}$ \\
\hline $\begin{array}{l}\text { Bart J. Emmer, } \\
\text { MD, PhD }\end{array}$ & $\begin{array}{l}\text { Amsterdam UMC, } \\
\text { University of } \\
\text { Amsterdam }\end{array}$ & $\begin{array}{l}\text { Critical revision of the } \\
\text { manuscript for intellectual } \\
\text { content }\end{array}$ \\
\hline $\begin{array}{l}\text { Rogier V. } \\
\text { Immink, MD, } \\
\text { PhD }\end{array}$ & $\begin{array}{l}\text { Amsterdam UMC, } \\
\text { University of } \\
\text { Amsterdam }\end{array}$ & $\begin{array}{l}\text { Critical revision of the } \\
\text { manuscript for intellectual } \\
\text { content }\end{array}$ \\
\hline $\begin{array}{l}\text { Charles B.L.M. } \\
\text { Majoie, MD, PhD }\end{array}$ & $\begin{array}{l}\text { Amsterdam UMC, } \\
\text { University of } \\
\text { Amsterdam }\end{array}$ & $\begin{array}{l}\text { Critical revision of the } \\
\text { manuscript for intellectual } \\
\text { content }\end{array}$ \\
\hline $\begin{array}{l}\text { Wim H. van } \\
\text { Zwam, MD, PhD }\end{array}$ & $\begin{array}{l}\text { Maastricht } \\
\text { University Medical } \\
\text { Center }\end{array}$ & $\begin{array}{l}\text { Critical revision of the } \\
\text { manuscript for intellectual } \\
\text { content }\end{array}$ \\
\hline $\begin{array}{l}\text { Reinoud P.H. } \\
\text { Bokkers, MD, } \\
\text { PhD }\end{array}$ & $\begin{array}{l}\text { University Medical } \\
\text { Center Groningen }\end{array}$ & $\begin{array}{l}\text { Critical revision of the } \\
\text { manuscript for intellectual } \\
\text { content }\end{array}$ \\
\hline
\end{tabular}

Appendix 1 (continued)

\begin{tabular}{|c|c|c|}
\hline Name & Location & Contribution \\
\hline $\begin{array}{l}\text { Maarten } \\
\text { Uyttenboogaart, } \\
\text { MD, PhD }\end{array}$ & $\begin{array}{l}\text { University Medical } \\
\text { Center Groningen }\end{array}$ & $\begin{array}{l}\text { Critical revision of the } \\
\text { manuscript for intellectual } \\
\text { content }\end{array}$ \\
\hline $\begin{array}{l}\text { Boudewijn } \\
\text { A.A.M. van } \\
\text { Hasselt, MD }\end{array}$ & $\begin{array}{l}\text { Isala hospital, } \\
\text { Zwolle }\end{array}$ & $\begin{array}{l}\text { Acquisition of data, critical } \\
\text { revision of the manuscript } \\
\text { for intellectual content }\end{array}$ \\
\hline $\begin{array}{l}\text { Jörg Mühling, } \\
\text { MD, PhD }\end{array}$ & $\begin{array}{l}\text { Radboud University } \\
\text { Medical Center, } \\
\text { Nijmegen }\end{array}$ & $\begin{array}{l}\text { Acquisition of data, critical } \\
\text { revision of the manuscript } \\
\text { for intellectual content }\end{array}$ \\
\hline $\begin{array}{l}\text { James F. Burke, } \\
\text { MD, PhD }\end{array}$ & $\begin{array}{l}\text { University of } \\
\text { Michigan, Ann } \\
\text { Arbor }\end{array}$ & $\begin{array}{l}\text { Critical interpretation of the } \\
\text { data and revision of the } \\
\text { manuscript for intellectual } \\
\text { content }\end{array}$ \\
\hline $\begin{array}{l}\text { Bob Roozenbeek, } \\
\text { MD, PhD }\end{array}$ & $\begin{array}{l}\text { Erasmus MC } \\
\text { University Medical } \\
\text { Center, Rotterdam }\end{array}$ & $\begin{array}{l}\text { Critical revision of the } \\
\text { manuscript for intellectual } \\
\text { content }\end{array}$ \\
\hline $\begin{array}{l}\text { Aad van der Lugt, } \\
\text { MD, PhD }\end{array}$ & $\begin{array}{l}\text { Erasmus MC } \\
\text { University Medical } \\
\text { Center, Rotterdam }\end{array}$ & $\begin{array}{l}\text { Study concept and design, } \\
\text { critical revision of the } \\
\text { manuscript for intellectual } \\
\text { content }\end{array}$ \\
\hline $\begin{array}{l}\text { Diederik W.J. } \\
\text { Dippel, MD, PhD }\end{array}$ & $\begin{array}{l}\text { Erasmus MC } \\
\text { University Medical } \\
\text { Center, Rotterdam }\end{array}$ & $\begin{array}{l}\text { Study concept and design, } \\
\text { interpretation of the data, } \\
\text { critical revision of the } \\
\text { manuscript for intellectual } \\
\text { content }\end{array}$ \\
\hline $\begin{array}{l}\text { Hester F. } \\
\text { Lingsma, PhD }\end{array}$ & $\begin{array}{l}\text { Erasmus MC } \\
\text { University Medical } \\
\text { Center, Rotterdam }\end{array}$ & $\begin{array}{l}\text { Study concept and design, } \\
\text { statistical analyses, } \\
\text { interpretation of the data, } \\
\text { critical revision of the } \\
\text { manuscript for intellectual } \\
\text { content }\end{array}$ \\
\hline $\begin{array}{l}\text { Adriaan C.G.M. } \\
\text { van Es, MD, PhD }\end{array}$ & $\begin{array}{l}\text { Erasmus MC } \\
\text { University Medical } \\
\text { Center, Rotterdam }\end{array}$ & $\begin{array}{l}\text { Study concept and design, } \\
\text { interpretation of the data, } \\
\text { critical revision of the } \\
\text { manuscript for intellectual } \\
\text { content }\end{array}$ \\
\hline
\end{tabular}

Appendix 2 Coinvestigators

Coinvestigators are listed at links.Iww.com/WNL/B252

\section{References}

1. Berkhemer OA, van den Berg LA, Fransen PS, et al. The effect of anesthetic management during intra-arterial therapy for acute stroke in MR CLEAN. Neurology 2016;87:656-664.

2. Campbell BCV, van Zwam WH, Goyal M, et al. Effect of general anaesthesia on functional outcome in patients with anterior circulation ischaemic stroke having endovascular thrombectomy versus standard care: a meta-analysis of individual patient data. Lancet Neurol 2018;17:47-53.

3. van de Graaf RA, Samuels N, Mulder M, et al. Conscious sedation or local anesthesia during endovascular treatment for acute ischemic stroke. Neurology 2018;91:e19-e25.

4. Goldhoorn RB, Bernsen MLE, Hofmeijer J, et al. Anesthetic management during endovascular treatment of acute ischemic stroke in the MR CLEAN registry. Neurology 2020;94:e97-e106.

5. Davis MJ, Menon BK, Baghirzada LB, et al. Anesthetic management and outcome in patients during endovascular therapy for acute stroke. Anesthesiology 2012;116: 396-405.

6. Lowhagen Henden P, Rentzos A, Karlsson JE, et al. Hypotension during endovascular treatment of ischemic stroke is a risk factor for poor neurological outcome. Stroke $2015 ; 46: 2678-2680$ 
7. Petersen NH, Ortega-Gutierrez S, Wang A, et al. Decreases in blood pressure during thrombectomy are associated with larger infarct volumes and worse functional outcome. Stroke 2019;50:1797-1804.

8. Treurniet KM, Berkhemer OA, Immink RV, et al. A decrease in blood pressure is associated with unfavorable outcome in patients undergoing thrombectomy under general anesthesia. J Neurointerv Surg 2018;10:107-111.

9. Pikija S, Trkulja V, Ramesmayer C, et al. Higher blood pressure during endovascular thrombectomy in anterior circulation stroke is associated with better outcomes. J Stroke 2018;20:373-384.

10. Devlin JW, Mallow-Corbett S, Riker RR. Adverse drug events associated with the use of analgesics, sedatives, and antipsychotics in the intensive care unit. Crit Care Med 2010;38:S231-S243.

11. Hug CC Jr, McLeskey CH, Nahrwold ML, et al. Hemodynamic effects of propofol: data from over 25,000 patients. Anesth Analg 1993;77:S21-S29.

12. Maier B, Fahed R, Khoury N, et al. Association of blood pressure during thrombectomy for acute ischemic stroke with functional outcome: a systematic review. Stroke 2019;50:2805-2812.

13. Alcaraz G, Chui J, Schaafsma J, et al. Hemodynamic management of patients during endovascular treatment of acute ischemic stroke under conscious sedation: a retrospective cohort study. J Neurosurg Anesthesiol 2019;31:299-305.

14. Whalin MK, Halenda KM, Haussen DC, et al. Even small decreases in blood pressure during conscious sedation affect clinical outcome after stroke thrombectomy: an analysis of hemodynamic thresholds. AJNR Am J Neuroradiol 2017;38:294-298.

15. Jansen IGH, Mulder M, Goldhoorn RB; investigators MCR. Endovascular treatment for acute ischaemic stroke in routine clinical practice: prospective, observational cohort study (MR CLEAN Registry). Bmj 2018;360:k949.

16. von Elm E, Altman DG, Egger M, et al. The strengthening the reporting of observational studies in Epidemiology (STROBE) statement: guidelines for reporting observational studies. Int J Surg 2014;12:1495-1499.

17. Bijker JB, van Klei WA, Kappen TH, van Wolfswinkel L, Moons KG, Kalkman CJ. Incidence of intraoperative hypotension as a function of the chosen definition: literature definitions applied to a retrospective cohort using automated data collection. Anesthesiology 2007;107:213-220.

18. Whalin MK, Halenda KM, Haussen DC, et al. Even small decreases in blood pressure during conscious sedation affect clinical outcome after stroke thrombectomy: an analysis of hemodynamic thresholds. Am J Neuroradiol 2017;38:294-298.

19. Valent A, Sajadhoussen A, Maier B, et al. A $10 \%$ blood pressure drop from baseline during mechanical thrombectomy for stroke is strongly associated with worse neurological outcomes. J Neurointerv Surg 2020;12:363-369.
20. Monk TG, Bronsert MR, Henderson WG, et al. Association between intraoperative hypotension and hypertension and 30-day postoperative mortality in noncardiac surgery. Anesthesiology 2015;123:307-319.

21. Talke PO, Sharma D, Heyer EJ, Bergese SD, Blackham KA, Stevens RD. Society for Neuroscience in Anesthesiology and Critical Care Expert Consensus Statement: Anesthetic Management of Endovascular Treatment for Acute Ischemic Stroke: endorsed by the Society of NeuroInterventional Surgery and the Neurocritical Care Society. J Neurosurg Anesthesiol 2014;26:95-108.

22. van Swieten JC, Koudstaal PJ, Visser MC, Schouten HJ, van Gijn J. Interobserver agreement for the assessment of handicap in stroke patients. Stroke 1988;19: 604-607.

23. Brott T, Adams HP Jr, Olinger CP, et al. Measurements of acute cerebral infarction: a clinical examination scale. Stroke 1989;20:864-870.

24. Goyal M, Fargen KM, Turk AS, et al. 2C or not 2C: defining an improved revascularization grading scale and the need for standardization of angiography outcomes in stroke trials. J Neurointerv Surg 2014;6:83-86.

25. von Kummer R, Broderick JP, Campbell BC, et al. The Heidelberg bleeding classification: classification of bleeding events after ischemic stroke and reperfusion therapy. Stroke 2015;46:2981-2986.

26. Donders AR, van der Heijden GJ, Stijnen T, Moons KG. Review: a gentle introduction to imputation of missing values. J Clin Epidemiol 2006;59:1087-1091.

27. Petersen NH, Silverman A, Kimmel AC, Sheth KN. Response by Petersen et al to letter regarding article, "Decreases in blood pressure during thrombectomy are associated with larger infarct volumes and worse functional outcome." Stroke 2019;50: e321-e322.

28. Rasmussen M, Schonenberger S, Henden PL, et al. Blood pressure thresholds and neurologic outcomes after endovascular therapy for acute ischemic stroke: an analysis of individual patient data from 3 randomized clinical trials. JAMA Neurol 2020;77: 622-631.

29. Schonenberger S, Uhlmann L, Hacke W, et al. Effect of conscious sedation vs general anesthesia on early neurological improvement among patients with ischemic stroke undergoing endovascular thrombectomy: a randomized clinical trial. JAMA 2016;316: 1986-1996.

30. Lowhagen Henden P, Rentzos A, Karlsson JE, et al. General anesthesia versus conscious sedation for endovascular treatment of acute ischemic stroke: the AnStroke Trial (Anesthesia During Stroke). Stroke 2017;48:1601-1607.

31. Simonsen CZ, Yoo AJ, Sorensen LH, et al. Effect of general anesthesia and conscious sedation during endovascular therapy on infarct growth and clinical outcomes in acute ischemic stroke: a randomized clinical trial. JAMA Neurol 2018;75:470-477. 


\section{Neurology}

\section{Blood Pressure During Endovascular Treatment Under Conscious Sedation or Local Anesthesia}

Noor Samuels, Rob A. van de Graaf, Carlijn A.L. van den Berg, et al. Neurology 2021;96; e171-e181 Published Online before print October 7, 2020

DOI 10.1212/WNL.0000000000011006

This information is current as of October 7, 2020

\section{Updated Information \& Services}

References

Subspecialty Collections

Permissions \& Licensing

Reprints including high resolution figures, can be found at: http://n.neurology.org/content/96/2/e171.full

This article cites 31 articles, 13 of which you can access for free at: http://n.neurology.org/content/96/2/e171.full\#ref-list-1

This article, along with others on similar topics, appears in the following collection(s):

\section{All Cerebrovascular disease/Stroke}

http://n.neurology.org/cgi/collection/all_cerebrovascular_disease_strok e

Clinical trials Observational study (Cohort, Case control)

http://n.neurology.org/cgi/collection/clinical_trials_observational_stud y_cohort_case_control

Information about reproducing this article in parts (figures,tables) or in its entirety can be found online at:

http://www.neurology.org/about/about_the_journal\#permissions

Information about ordering reprints can be found online:

http://n.neurology.org/subscribers/advertise

Neurology ${ }^{\circledR}$ is the official journal of the American Academy of Neurology. Published continuously since 1951, it is now a weekly with 48 issues per year. Copyright Copyright ( 2020 The Author(s). Published by Wolters Kluwer Health, Inc. on behalf of the American Academy of Neurology.. All rights reserved. Print ISSN: 0028-3878. Online ISSN: 1526-632X.

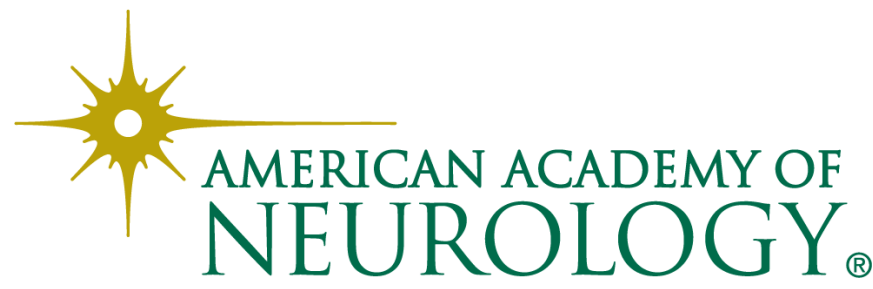

